\title{
Photoreaction Dynamics of Red-Shifting Retinal Analogues Reconstituted in Proteorhodopsin
}

\author{
Yusaku Hontani, ${ }^{\dagger}$ Srividya Ganapathy, ${ }^{\ddagger \odot}$ Sean Frehan, ${ }^{\dagger}$ Miroslav Kloz, ${ }^{\S}$ Willem J. de Grip, ${ }^{\ddagger}, \|_{\odot}$ \\ and John T.M. Kennis* ${ }^{* \dagger \odot}$ \\ ${ }^{\dagger}$ Department of Physics and Astronomy, Vrije Universiteit, Amsterdam $1081 \mathrm{HV}$, The Netherlands \\ ${ }^{\ddagger}$ Department of Biophysical Organic Chemistry, Leiden Institute of Chemistry, Gorlaeus Laboratories, Leiden University, Leiden \\ 2300 RA, The Netherlands \\ ${ }^{\S}$ ELI-Beamlines, Institute of Physics, Na Slovance 2, Praha 8182 21, Czech Republic \\ "Department of Biochemistry, Radboud University Medical Center, Nijmegen 6500 HB, The Netherlands
}

Supporting Information

\begin{abstract}
Microbial rhodopsins constitute a key protein family in optobiotechnological applications such as optogenetics and voltage imaging. Spectral tuning of rhodopsins into the deep-red and near-infrared spectral regions is of great demand in such applications because more bathochromic light into the near-infrared range penetrates deeper in living tissue. Recently, retinal analogues have been successfully used in ion transporting and fluorescent rhodopsins to achieve red-shifted absorption, activity, and emission properties. Understanding their photochemical mechanism is essential for further design
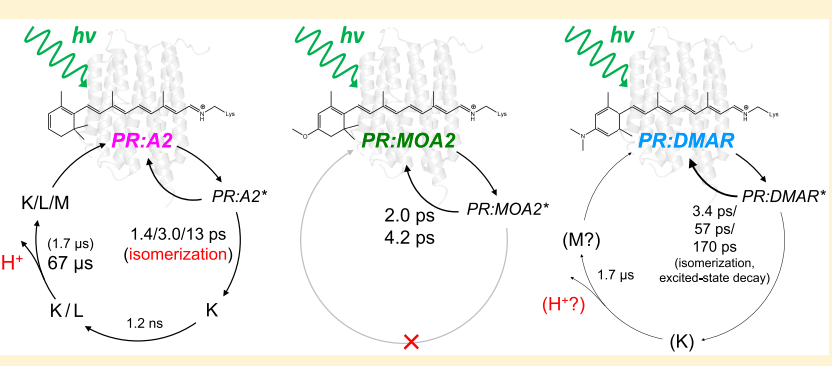
of appropriate retinal analogues but is yet only poorly understood for most retinal analogue pigments. Here, we report the photoreaction dynamics of red-shifted analogue pigments of the proton pump proteorhodopsin (PR) containing A2 (all-trans3,4-dehydroretinal), MOA2 (all-trans-3-methoxy-3,4-dehydroretinal), or DMAR (all-trans-3-dimethylamino-16-nor-1,2,3,4didehydroretinal), utilizing femto- to submillisecond transient absorption spectroscopy. We found that the A2 analogue photoisomerizes in 1.4, 3.0, and/or 13 ps upon $510 \mathrm{~nm}$ light illumination, which is comparable to the native retinal (A1) in PR. On the other hand, the deprotonation of the A2 pigment Schiff base was observed with a dominant time constant of $67 \mu$ s, which is significantly slower than the A1 pigment. In the MOA2 pigment, no isomerization or photoproduct formation was detected upon $520 \mathrm{~nm}$ excitation, implying that all the excited molecules returned to the initial ground state in 2.0 and 4.2 ps. The DMAR pigment showed very slow excited state dynamics similar to the previously studied MMAR pigment, but only very little photoproduct was formed. The low efficiency of the photoproduct formation likely is the reason why DMAR analogue pigments of PR showed very weak proton pumping activity.
\end{abstract}

\section{INTRODUCTION}

Rhodopsins are essential photoreceptor proteins present in many organisms and display a variety of functionalities. ${ }^{1,2}$ Rhodopsins are generally classified into two groups: microbial (type I) and animal (type II) rhodopsins. ${ }^{1}$ Both types incorporate a retinal chromophore absorbing visible light (retinal A1). Microbial rhodopsins, in particular the lightdriven ion transporters, have been widely used in optobiotechnological applications such as optogenetics ${ }^{3-6}$ and fluorescence-based voltage imaging. ${ }^{7-9}$

Spectral tuning of rhodopsins into the deep-red and nearinfrared spectral regions is of great demand for such applications because these photons can penetrate deeper in living tissue ${ }^{10}$ so that deep tissue application becomes feasible. The extent of the $\pi$-electron conjugation of the chromophore and the degree of electron delocalization are key factors in setting the position of the absorption band and absorption maximum. Recently, significant spectral red-shifting was achieved in proton pumps, cation channels, and fluorescent microbial rhodopsins using retinal analogues having extended $\pi$-electron-conjugated systems. ${ }^{1-16}$ Importantly, some of these retinal analogues maintain the transport activity of their hosts. $^{12-14}$

Particularly, the A2 retinal analogue (all-trans-3,4-dehydroretinal), which is the only other naturally occurring retinal analogue and has additional $\pi$ conjugation between $\mathrm{C} 3$ and $\mathrm{C} 4$ on the $\beta$-ionone ring ${ }^{17-19}$ (Figure 1), exhibits a high affinity for the opsin protein because of the similar configuration to the native A1 retinal. Accordingly, the A2 analogue has been shown to induce a red shift in channelrhodopsins ${ }^{11}$ and proton pumping rhodopsins by $\sim 30-40 \mathrm{~nm}^{13,14}$ while nearly completely maintaining their ion transport activity. In addition,

Received: February 4, 2019

Revised: April 18, 2019

Published: April 18, 2019 

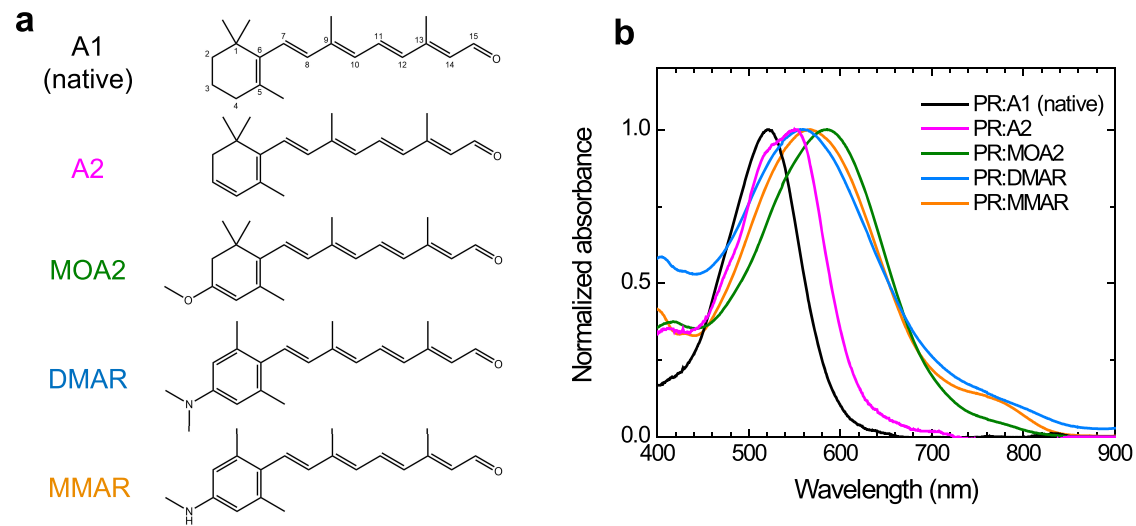

Figure 1. Chemical structures and steady-state absorption spectra. (a) Structures of the native retinal (A1) and retinal analogues (A2, MOA2, $\mathrm{DMAR}$, and MMAR) and (b) the main absorbance band of the corresponding analogue pigments generated with $\mathrm{PR}$, recorded at $\mathrm{pH}$. Data are based upon Ganapathy et al. 2017. ${ }^{14}$

the MOA2 retinal analogue (all-trans-3-methoxy-3,4-dehydroretinal, Figure 1), which has additional $\pi$ conjugation between $\mathrm{C} 3$ and $\mathrm{C} 4$ and, in addition, the strong electron-withdrawing methoxy group at position $\mathrm{C} 3$ in the $\beta$-ionone ring, has been shown to induce very large red shifts $(65-80 \mathrm{~nm})$ compared with the native $\mathrm{A} 1$ in proton pumping rhodopsins. ${ }^{14}$ Although the proton pump function in the MOA2 pigment was only clearly detected under white light illumination, ${ }^{14}$ MOA2 might be an interesting candidate for deep-red tuning of other rhodopsin proteins. Furthermore, the DMAR retinal analogue (all-trans-3-dimethylamino-16-nor-1,2,3,4-didehydroretinal, Figure 1), which has an aromatized ring moiety with a dimethylamino substituent at $\mathrm{C} 3$, is another promising candidate for red-tuning of microbial rhodopsins. For instance, incorporation of DMAR in the proton pump proteorhodopsin (PR) yielded a $\sim 40 \mathrm{~nm}$ red shift relative to $\mathrm{Al}$, but only weak proton pump activity was observed. ${ }^{14}$ On the other hand, in the H134R and H134R/T159C variants of the channelrhodopsin ChR2, which are widely used in optogenetics, ${ }^{20}$ incorporation of a related retinal analogue, containing an aromatized ring with a dimethylamino group, but lacking the methyl groups on the ring, resulted in a $\sim 40 \mathrm{~nm}$ red shift while partially maintaining the transport function. ${ }^{12}$

Understanding of the photochemical mechanism of rhodopsins containing retinal analogues is essential for further development and design of functional retinal analogue pigments. Typically, in ion-pumping microbial rhodopsins, the photoreaction in the chromophore proceeds in the time range of femtoseconds to milliseconds. ${ }^{1}$ Hence, observation of this time range is fundamental for understanding the photochemistry. In our previous work, the photochemistry of the 3-methylamino-16-nor-1,2,3,4-didehydroretinal analogue pigment of PR (PR:MMAR) was clarified with femto- to submillisecond transient absorption spectroscopy, showing unusually long-lasting excited states and a very high fluorescence quantum yield. ${ }^{21}$ However, for other retinal analogue pigments, experimental data are very limited. In this study, we applied femtosecond to submillisecond transient absorption spectroscopy to $\mathrm{PR}$ analogue pigments containing A2 (PR:A2), MOA2 (PR:MOA2), or DMAR (PR:DMAR) analogues in order to investigate the excited state and groundstate photoproduct dynamics. Our results reveal significant differences in photodynamic behavior of these three analogue pigments, which show a correlation with their functional activity.

\section{METHODS}

Protein Purification. All-trans retinal (hereafter called A1) was obtained from Sigma-Aldrich; all-trans-3,4-dehydroretinal (A2) was a generous gift from Hoffman-LaRoche. All-trans-3methoxy-3,4-dehydroretinal (MOA2; purity 97.8\% according to manufacturer's certificate of analysis) and all-trans-3dimethylamino-16-nor-1,2,3,4-didehydroretinal (DMAR; purity $99.9 \%$ ) were synthesized on order by Buchem, B.V. Escherichia coli strain UT5600 transformed with the pKJ900 plasmid was used to express recombinant PR with a C-terminal 6-His tag. The cells were grown in Lysogeny broth medium, harvested, lysed, and regenerated with the appropriate retinal as per protocols described previously. ${ }^{13,14}$ The lysed cell suspension was incubated in $4 \% \operatorname{DDM}(\mathrm{w} / \mathrm{v})$ overnight at 4 ${ }^{\circ} \mathrm{C}$ and centrifuged to remove the insoluble cellular debris. The His-tagged PRs were purified by immobilized metal affinity chromatography, as reported previously. ${ }^{14} \mathrm{Ni}^{2+}-\mathrm{NTA}$ resin (Qiagen, $10 \mathrm{~mL}$ ) was used per $2000 \mathrm{~mL}$ original culture volume. The purified $\mathrm{PR}$ was eluted in $5 \mathrm{~mL}$ fractions containing $20 \mathrm{mM}$ bis-Tris propane, $500 \mathrm{mM}$ imidazole, 0.5 $\mathrm{M} \mathrm{NaCl}, 0.1 \%$ DDM, pH 8 at room temperature. The fractions were combined and concentrated using a $10 \mathrm{kDa}$ cut-off column (Amicon) to $\mathrm{OD} \approx 10$ or $\sim 20$ at the absorption maxima for transient absorption spectroscopy and steady-statestimulated Raman spectroscopy, respectively. Buffer solutions for $\mathrm{pH}$-dependent experiments contained $150 \mathrm{mM} \mathrm{NaCl}$ and $2.5 \% \mathrm{DDM}$, with $20 \mathrm{mM}$ bis-Tris propane for $\mathrm{pH} 7.0$ or 20 $\mathrm{mM}$ Tris- $\mathrm{HCl}$ for $\mathrm{pH} 9.0$.

Steady-State-Stimulated Raman Spectroscopy. Steady-state-stimulated Raman experiments were performed as reported previously using a home-built-stimulated Raman setup with an $800 \mathrm{~nm}$ Raman pump. ${ }^{22-24}$ The protein samples were filled in a $2 \mathrm{~mm}$ pathlength quartz cuvette (100-QS, Hellma Analytics).

Femto- to Submillisecond Transient Absorption Spectroscopy. Transient absorption measurements were performed with a home-built femtosecond to submillisecond pump-probe setup as reported previously. ${ }^{23,25,26}$ The samples were filled in a homemade sample holder that has two $2 \mathrm{~mm}$ thick $\mathrm{CaF}_{2}$ plates. The sample thickness was set at $400 \mu \mathrm{m}$ for transient absorption experiments with an appropriate sample spacer. The sample holder was set on a Lissajous scanner that ensures sample refreshment after each laser shot with a time interval of $60 \mathrm{~s}$ between successive exposures to the laser 
pulses. $^{27} \mathrm{~A} \mathrm{CaF}_{2}$ plate on a moving stage was used for supercontinuum white light generation, and a selected wavelength region of $400-750 \mathrm{~nm}$ was detected by the photodiode array. The polarization between the pump and probe pulses was set at the magic angle $\left(\sim 54.7^{\circ}\right)$. The time delay varied up to $400 \mu \mathrm{s}$ at 163 data points with a minimum temporal step of $50 \mathrm{fs}$. The diameters of the pump and the probe beams at the sample position were $\sim 200$ and $\sim 50 \mu \mathrm{m}$ (full width at half-maximum), respectively. The central wavelength and the power of the pump beam were set at $510 \mathrm{~nm}(\sim 400 \mathrm{~nJ})$ and $620 \mathrm{~nm}(\sim 600 \mathrm{~nJ})$ for PR:A2, at 520 $\mathrm{nm}(\sim 500 \mathrm{~nJ})$ for PR:MOA2, and at $500 \mathrm{~nm}(\sim 600 \mathrm{~nJ})$ and $600 \mathrm{~nm}(\sim 600 \mathrm{~nJ})$ for PR:DMAR. The instrumental response function was $\sim 100 \mathrm{fs}$, as estimated from global analysis.

Global Analysis Methodology. Global analysis was performed for the transient absorption spectra using the Glotaran program. ${ }^{28}$ With global analysis, all wavelengths/ wavenumbers were analyzed simultaneously with a set of common time constants. ${ }^{29}$ A kinetic model was applied consisting of sequentially interconverting, evolution-associated difference spectra (EADS), that is, $1 \rightarrow 2 \rightarrow 3 \rightarrow \ldots$, in which the arrows indicate successive monoexponential decays of a time constant, which can be regarded as the lifetime of each EADS. ${ }^{29}$ The first EADS corresponds to the difference spectrum at time zero. The first EADS evolves into the second EADS with time constant $\tau_{1}$, which in turn evolves into the third EADS with time constant $\tau_{2}$, and so forth. The procedure clearly visualizes the evolution of the intermediate states of the protein. $^{23,25,30,31}$ Decay-associated difference spectra (DADS) indicate the spectral changes with parallel decay channels and independent decay time constants. Intuitively, the DADS provide the difference spectra between the subsequent EADS, provided that the time constants are sufficiently widely separated. It is important to note that the parallel and the sequential analysis are mathematically equivalent and yield identical time constants. ${ }^{29,32}$ The standard errors in the time constants were less than $5 \%$.

\section{RESULTS AND DISCUSSION}

Photochemistry of PR:A2. First, we investigate the ground-state retinal configuration of the A2 chromophore in PR. As seen in Figure 1, PR:A2 shows two main absorption bands at $\sim 552$ and $\sim 515 \mathrm{~nm}$, while the native pigment (PR:A1) has a single band at $\sim 520 \mathrm{~nm}$. This observation implies that PR:A2 might have heterogeneous ground-state conformers. Therefore, preresonance-watermarked stimulated Raman spectroscopy with an $800 \mathrm{~nm}$ Raman pump ${ }^{21-24}$ was applied to PR:A2 at a physiological $\mathrm{pH}(7.0)$ to investigate the ground-state characteristics of the chromophore (Figure 2, magenta line). Note that our previous $\mathrm{pH}$ titration study showed that the pKa of PR:A2 is $\sim 6.2$ in DDM solution; ${ }^{14}$ thus, mostly PR:A2 is in the alkaline form. The Raman spectrum of PR:A2 is quite similar to that of PR:A1, ${ }^{33,34}$ except for a strong additional band at $1512 \mathrm{~cm}^{-1}$. In the $\mathrm{C}-\mathrm{C}$ stretching region, peaks are observed at 1171, 1182, 1200, 1238 , and $1253 \mathrm{~cm}^{-1}$. These peaks correspond to Raman peaks of the all-trans A1 chromophore in BR and PR (1170/1172, $1187 / 1185,1201 / 1198,1234$, and $\left.1255 / 1253 \mathrm{~cm}^{-1}\right)^{33-36}$ (corresponding to $1168,1185,1199,1236$, and $1252 \mathrm{~cm}^{-1}$, respectively, in our Raman spectra of PR:A1 Figure 2, black line). The single peak at $1656 \mathrm{~cm}^{-1}$, which is assigned to the $\mathrm{C}=\mathrm{N}$ stretch vibration of the protonated retinylidene Schiff base (RSB), and the absence of a strong peak near $800 \mathrm{~cm}^{-1}$,

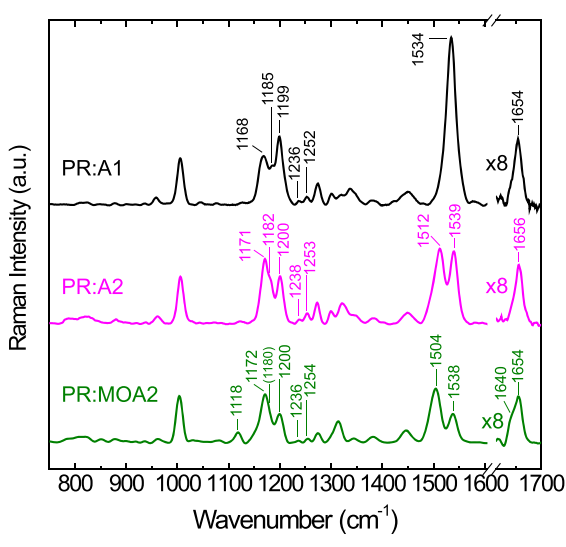

Figure 2. Ground-state-stimulated Raman spectrum of PR:A1, PR:A2, and PR:MOA2 obtained with an $800 \mathrm{~nm}$ Raman pump. $\mathrm{pH}$ was set at 7.0 for PR:A1 and PR:A2, and 9.0 for PR:MOA2. The $y$-axis in the spectral region between 1610 and $1700 \mathrm{~cm}^{-1}$ is scaled up 8 -fold. The spectra were normalized upon the $1010 \mathrm{~cm}^{-1}$ band.

which is characteristic for the 13-cis configuration, ${ }^{37}$ also agree with an all-trans configuration of the A2 chromophore. Although a minor amount of 13-cis species may be present, represented by a small $1185 \mathrm{~cm}^{-1}$ peak,,$^{37,38}$ there is general consensus that in the ground state, PR:A1 contains an all-trans chromophore, $33,36,38,39$ and we conclude that the same is true for PR:A2. In the $\mathrm{C}=\mathrm{C}$ stretching region of PR:A2, two large peaks at 1539 and $1512 \mathrm{~cm}^{-1}$ are present. This could reflect ground-state heterogeneity in PR:A2, which for instance could express itself in the pluriform absorption spectrum (cf. Figure 1b). It should be noted, however, that the $1512 \mathrm{~cm}^{-1}$ band relatively poorly matches the corresponding absorption maximum at $552 \mathrm{~nm}$, when compared to the established relation between these quantities. ${ }^{40-43}$

Next, the photochemistry of PR:A2 was investigated at $\mathrm{pH}$ 7.0 by applying femto-to submillisecond transient absorption spectroscopy with $510 \mathrm{~nm}$ excitation. The transient absorption spectra were globally fitted with eight time components: $170 \mathrm{fs}$, $1.4 \mathrm{ps}, 3.0 \mathrm{ps}, 13 \mathrm{ps}, 1.3 \mathrm{~ns}, 1.7 \mu \mathrm{s}, 67 \mu \mathrm{s}$, and infinite. The EADS and DADS are shown in Figure $3 a, b$, and selected time traces are shown in Figure S1a. The first EADS (gray line, Figure $3 \mathrm{a}, \mathrm{b}$ ) evolves to the second EADS (red line) in $170 \mathrm{fs}$; observable are positive excited state absorption (ESA) at $\sim 610$ $\mathrm{nm}$ (strong) and $\sim 500 \mathrm{~nm}$ (weak), with negative stimulated emission (SE) at $>\sim 700 \mathrm{~nm}$, which confirms that the molecules are in the excited state. Notably, the red-shifted ESA was not seen in PR:A1, ${ }^{44,45}$ but a similar red-shifted ESA was reported in channelrhodopsins $\left(\mathrm{ChR} 2^{46,47}\right.$ and $\left.\mathrm{C} 1 \mathrm{C} 2^{25}\right)$. The negative ground-state bleach (GSB) signal is probably strongly overlapped with the ESA, thus not visible in the red EADS. In $1.4 \mathrm{ps,} \mathrm{development} \mathrm{to} \mathrm{the} \mathrm{third} \mathrm{EADS} \mathrm{(blue} \mathrm{in}$ Figure 3a,b) was observed, involving a large decay in the SE and ESA signals, while a small SE component still remained. The negative GSB signal at $\sim 550 \mathrm{~nm}$ became visible on the third EADS likely because the strong ESA decayed partially in 1.4 ps. After 3.0 and 13 ps evolution, the fifth EADS appears (magenta in Figure 3a), which no longer showed a SE band, indicating that the excited states were no longer populated after the 3.0 and 13 ps decay. Hence, it can be considered that the fifth EADS (magenta), which has an absorption peak at $\sim 610 \mathrm{~nm}$, shows the first K-like photoproduct state. The ESA at $\sim 620 \mathrm{~nm}$ strongly overlaps with the K-like absorption at $\sim 610 \mathrm{~nm}$. Thus, from the current transient absorption data, it 


\section{$510 \mathrm{~nm}$ excitation}

a

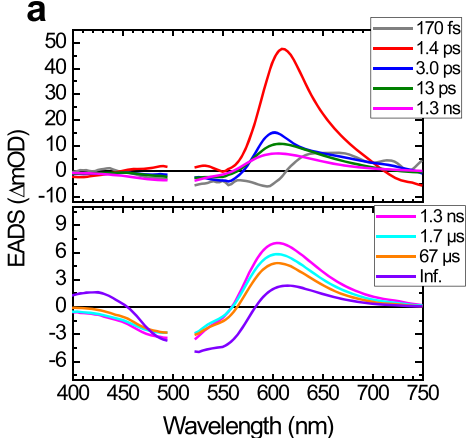

$\underline{620 \mathrm{~nm} \text { excitation }}$

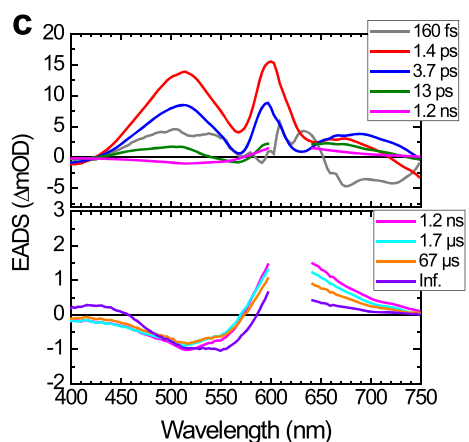

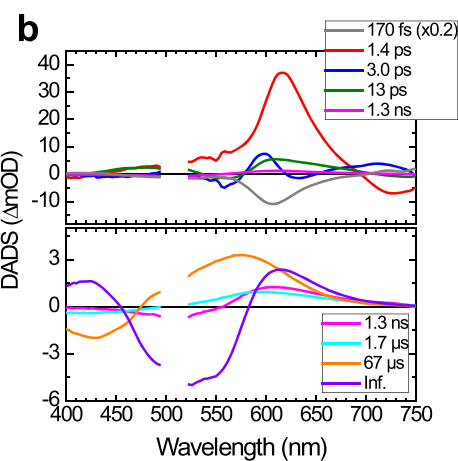

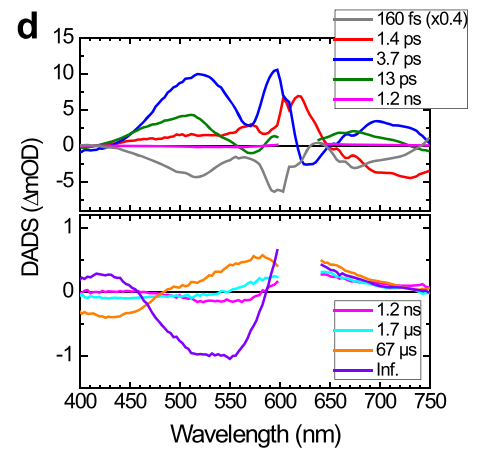

e

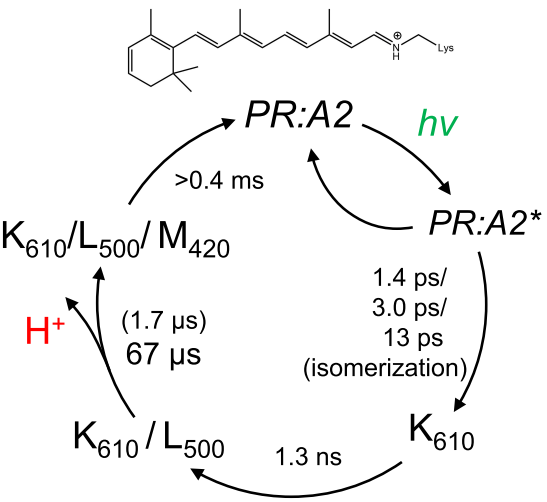

Figure 3. Photochemistry of PR:A2 at $\mathrm{pH} 7.0$. Globally fitted spectra upon $510 \mathrm{~nm}$ excitation; (a) EADS and (b) DADS. In the DADS, the 170 fs component is rescaled. Globally fitted spectra upon $620 \mathrm{~nm}$ excitation; (c) EADS and (d) DADS. In the DADS, the $160 \mathrm{fs}$ component is rescaled. The first five and the following components are shown in the top and the bottom panel, respectively. The wavelength regions of 500-520 nm in $(\mathrm{a}, \mathrm{b})$ and $600-635 \mathrm{~nm}$ in $(\mathrm{c}, \mathrm{d})$ are omitted because of the strong scattering. (e) Proposed photocycle model of PR:A2 upon excitation at $510 \mathrm{~nm}$.

is difficult to decide which one of the time components (1.4, 3.0 , and/or $13 \mathrm{ps})$ is involved in the all-trans to 13 -cis isomerization in PR:A2, and it also is difficult to identify the J$\mathrm{K}$ state transition which is assigned to vibrational cooling of the first photoproduct state. Using single-pulse or multipulse spectroscopy, it was reported that at acidic to neutral $\mathrm{pH}$, multiphasic isomerization in PR:A1 proceeds with three components $(0.2-0.7,6-10$, and $20-30 \mathrm{ps}) .^{44,45,48,49}$ Therefore, it seems like the photoisomerization of $\mathrm{A} 2$ in $\mathrm{PR}$ proceeds in a time frame similar to A1.

According to the DADS in Figure $3 \mathrm{~b}$ (bottom panel), in 1.3 $\mathrm{ns}$, the absorbance of $\mathrm{K}$ at $610 \mathrm{~nm}$ is decaying with a concomitant rise around $500 \mathrm{~nm}$. The $\sim 500 \mathrm{~nm}$ absorbance band can be assigned to an L-like intermediate. The K-like absorption largely persists after the $1.3 \mathrm{~ns}$ signal development, implying that a K/L equilibrium is formed in $1.3 \mathrm{~ns}$. In 1.7 and $67 \mu \mathrm{s}$, an absorbance band around $420 \mathrm{~nm}$ appears, which is assigned to an M-like intermediate, in which the RSB is deprotonated. It may be concluded that proton transfer from the RSB of A2 proceeds with 1.7 and $67 \mu$ s time components. From the DADS in Figure $3 \mathrm{~b}$ (bottom), the rise of the M-like intermediate appears to be $\sim 5$-fold larger in amplitude in $67 \mu \mathrm{s}$ than in $1.7 \mu \mathrm{s}$, which implies that the $67 \mu \mathrm{s}$ component is dominant for the proton transfer process. The K-intermediate absorption still persists after $67 \mu \mathrm{s}$, indicating that a $\mathrm{K} / \mathrm{L} / \mathrm{M}$ equilibrium, also observed in the chimeric channelrhodopsin $\mathrm{C} 1 \mathrm{C} 2,{ }^{25}$ is formed in $67 \mu \mathrm{s}$. For PR:A1, it was reported that at acidic to neutral $\mathrm{pH}$, the M-like intermediate is formed with components of $2-4 \mu \mathrm{s}$ and several tens of $\mu \mathrm{s}$. ${ }^{49,50}$ Hence, the dominant $\mathrm{M}$ formation in PR:A2 $(67 \mu \mathrm{s})$ seems to be somewhat slower than in PR:A1, while the minor $\mathrm{M}$ formation proceeds at almost the same rate in both pigments.

Because our steady-state absorption and Raman results suggest ground-state heterogeneity of PR:A2, we also applied $620 \mathrm{~nm}$ excitation to predominantly excite the $\sim 550 \mathrm{~nm}$ absorbing species. We observed a different set of ESA species peaking at $\sim 500,600$, and $670 \mathrm{~nm}$ (Figure 3c,d, top) but a similar set of photoproducts (Figure $3 \mathrm{~d}, \mathrm{c}$, bottom). Selected time traces are shown in Figure S1b. Significantly, the blueshifted ESA was also seen in the native PR:A1 ${ }^{44}$ and other microbial rhodopsins. ${ }^{23,30,51-53}$ The GSB includes a peak at $\sim 520 \mathrm{~nm}$ too, implying that a substantial part of the $\sim 520 \mathrm{~nm}$ species was also excited by the $620 \mathrm{~nm}$ pump. Likely, the ESA at $\sim 600$ and $\sim 670 \mathrm{~nm}$ upon $620 \mathrm{~nm}$ excitation results from the ESA as was shown above for $510 \mathrm{~nm}$ excitation (Figure 3a,b). The signal development of the photoproducts was almost identical for 620 and $510 \mathrm{~nm}$ excitations, but the signal intensity was significantly larger with $510 \mathrm{~nm}$ excitation. The molar absorption coefficient of PR:A2 at $510 \mathrm{~nm}$ is about 5.6fold higher than at $620 \mathrm{~nm}$ (Figure 1), the excitation power at $620 \mathrm{~nm}$ was 1.5 times that at $510 \mathrm{~nm}$ (see the Methods section), and with the same power, the photon flux at $620 \mathrm{~nm}$ is higher by a factor of 1.2. Therefore, we estimate that the ratio of the number of excited molecules is about 3.1:1 for 510 and $620 \mathrm{~nm}$ excitation, respectively. However, the Mintermediate population was about 4.8-fold stronger with 510 $\mathrm{nm}$ excitation (Figure $3 \mathrm{~b}, \mathrm{~d}$, bottom and Figure S1). Hence, this would indicate that $510 \mathrm{~nm}$ light can trigger the photocycle somewhat more efficiently than $620 \mathrm{~nm}$ light, implying that the hypsochromic ground-state species is somewhat more dominant in producing a functional photo- 

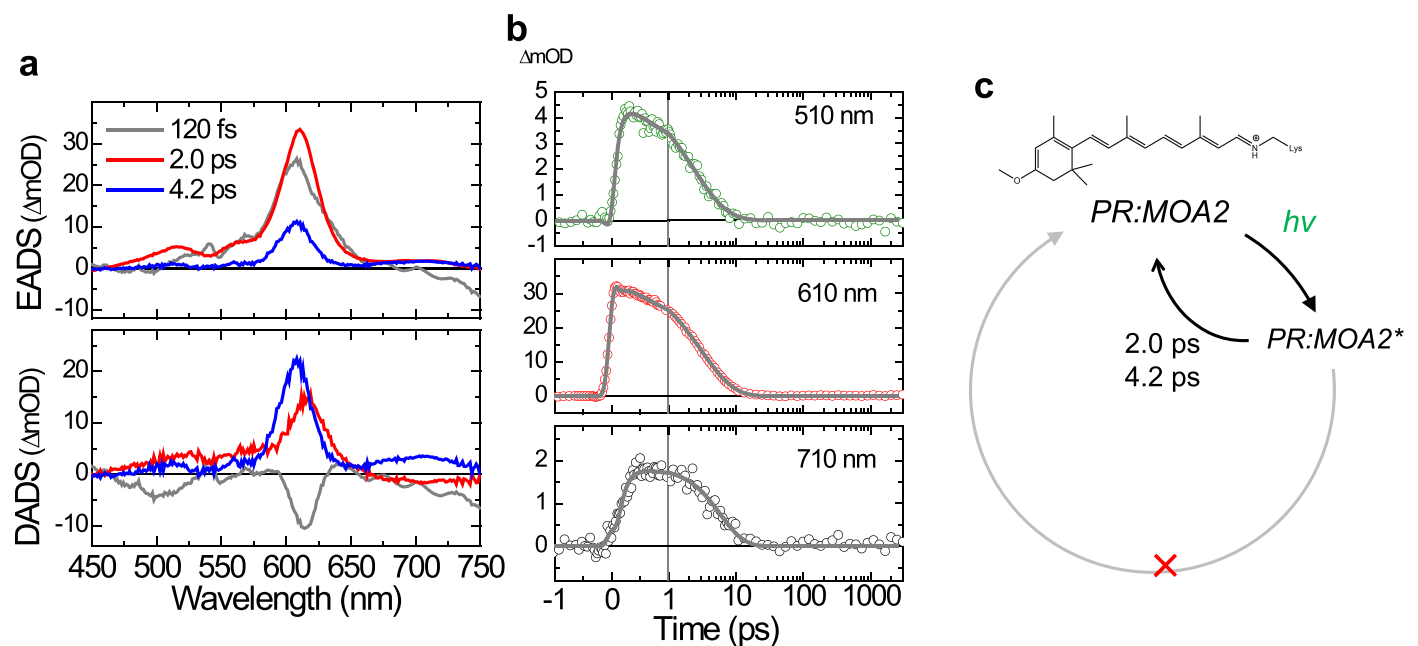

Figure 4. Transient absorption spectra of PR:MOA2 at pH 9.0 upon $520 \mathrm{~nm}$ excitation. (a) EADS (top) and DADS (bottom), (b) selected time traces at 510,610, and $710 \mathrm{~nm}$. Open dots show the raw data, and the solid lines show fitting curves. The time axis is linear until 1 ps and logarithmic thereafter. (c) Suggested photocycle of PR:MOA2 at pH 9.0 upon $520 \mathrm{~nm}$ excitation.
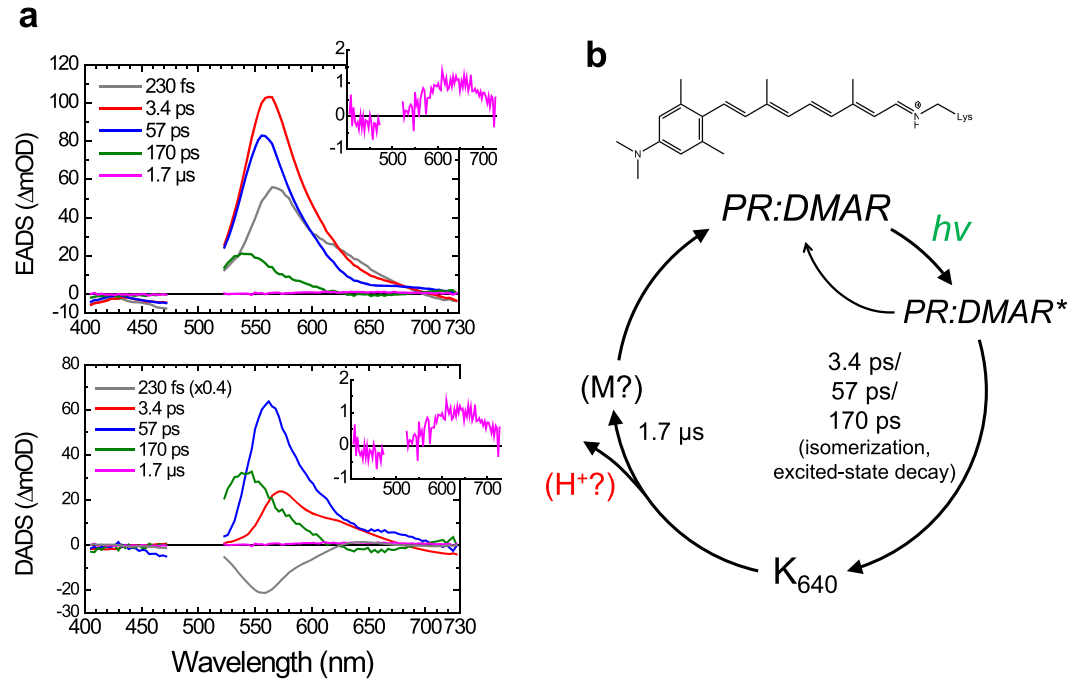

Figure 5. Photochemistry of PR:DMAR at $\mathrm{pH} 9.0$ upon $500 \mathrm{~nm}$ excitation. (a) EADS (top) and DADS (bottom). The $1.7 \mu$ s component (magenta line) is magnified in the inset. (b) Proposed photocycle model of PR:DMAR upon excitation at $500 \mathrm{~nm}$.

cycle in PR:A2. On the other hand, higher production of the M-intermediate with blue light under acidic conditions was also reported for PR:A1 ${ }^{50}$ and hence could simply be the result of populating higher energy vibronic states on the excited state surface. For PR:A1, it has been reported that the general scheme of the photocycle is similar at $\mathrm{pH} 7$ and $\mathrm{pH} 9$, be it that the kinetics and quantities of the photoproducts may vary. $33,44,48-50,54,55$

In Figure 3e, the photocycle of PR:A2 upon $510 \mathrm{~nm}$ excitation at $\mathrm{pH} 7.0$ is proposed. Obviously and not surprisingly, the photocycle bears great similarity to that of PR:A1. ${ }^{44,48-50}$

Photochemistry of PR:MOA2. The ground-state Raman spectrum of PR:MOA2 at $\mathrm{pH}$ 9.0, where the counterion Asp97 is fully deprotonated, ${ }^{14}$ is shown in Figure 2 (green line). Again, C-C stretching peaks were observed at 1172, 1180 (weak), 1200, 1236, and $1254 \mathrm{~cm}^{-1}$, but two $\mathrm{C}=\mathrm{NH}$ stretching peaks were seen at 1640 and $1654 \mathrm{~cm}^{-1}$. The most straightforward explanation is that the chromophore of PR:MOA2 adopts heterogeneous all-trans and 13-cis con- formers in the dark state. ${ }^{35,37}$ The stronger peak at $1118 \mathrm{~cm}^{-1}$ would also support the presence of a 13-cis chromophore. On the other hand, no significant peaks of isolated hydrogen outof-plane modes were observed in the $800-900 \mathrm{~cm}^{-1}$ region, which would reflect structurally distorted 13-cis conformers as observed in microbial rhodopsins containing 13-cis chromophores. $^{37,56}$ The two strong $\mathrm{C}=\mathrm{C}$ stretching peaks at 1504 and $1538 \mathrm{~cm}^{-1}$ further suggest that heterogeneous species exist in the dark state of MOA2, which could also explain the broad absorbance peak (Figure 1).

The photochemistry of PR:MOA2 was studied with $520 \mathrm{~nm}$ excitation at $\mathrm{pH}$ 9.0. Remarkably, only femtosecond and picosecond components were required in the global analysis, yielding time components of $120 \mathrm{fs}, 2.0$, and $4.2 \mathrm{ps}$ (Figure $4 \mathrm{a}, \mathrm{b})$. In $120 \mathrm{fs}$, a slight rise in a signal at $\sim 610 \mathrm{~nm}$ was observed. In the 2.0 and 4.2 ps components (red and blue lines in Figure 4a, respectively), strong positive signals were seen, but no negative peaks were detected. The multiple positive peaks are assigned to ESA bands with maxima at $\sim 520, \sim 560$, $\sim 610$, and $\sim 700 \mathrm{~nm}$. Probably, the GSB and SE signals 
strongly overlap with the ESA, explaining the absence of clear negative signals in the red and blue spectra in Figure 4a. Concomitantly, with the decay of the 4.2 ps component, all $\Delta A$ signals decayed completely (Figures $4 \mathrm{a}, \mathrm{b}$ and S2), implying that all excited molecules returned to the initial ground state in 4.2 ps. This observation indicates that under the applied conditions, PR:MOA2 does not generate detectable photoproducts, that is, photoisomerization does not seem to occur. Furthermore, the absence of M-like absorption in the near-UV region suggests that proton transfer from the protonated RSB in PR:MOA2 does not occur either. This would explain why hardly any pump activity is obtained upon illumination with small bandwidth light-emitting diode sources. ${ }^{14}$ However, continuous white light illumination does produce detectable proton pump activity. ${ }^{14}$ These observations are not easily reconciled. Our ground-state Raman data indicate heterogeneity in the Schiff base region, possibly due to the presence of all-trans as well as 13-cis conformers in the chromophore. We cannot exclude that this complex system reacts differently to continuous white light illumination than to femtosecond laser light excitation at $520 \mathrm{~nm}$. In Figure 4c, we present the photocycle of PR:MOA2 as deduced from our $520 \mathrm{~nm}$ excitation data.

Photochemistry of PR:DMAR. The photochemistry of PR:DMAR was studied at $\mathrm{pH} 9.0$ with 500 and $600 \mathrm{~nm}$ excitations. In the previous steady-state preresonance Raman study of PR:MMAR, ${ }^{21}$ the data support an all-trans configuration for the chromophore. Considering the strong structural similarity of DMAR and MMAR, we tentatively assume that this is also the case for PR:DMAR. The fs $-\mu \mathrm{s}$ transient absorption data upon $500 \mathrm{~nm}$ excitation were globally fitted with five components: $230 \mathrm{fs}, 3.4,57,170 \mathrm{ps}$, and $1.7 \mu \mathrm{s}$ (Figure 5a). The negative signals at $<\sim 480 \mathrm{~nm}$ seen in all transient absorption spectra are assigned to GSB. The ESA on the first four components is strongly overlapped with GSB and likely SE. The second EADS (red line, Figure 5a) has a negative signal at $>700 \mathrm{~nm}$, which is assigned to SE. Hence, the excited state was still populated after the first $220 \mathrm{fs}$ decay. The third and fourth EADS have no or only a slight negative signal at $>700 \mathrm{~nm}$. This observation may indicate that the excited state was depopulated in 3.4 and/or 57 ps. Alternatively, the negative SE signals were not apparent because of the strong overlap with ESA, and the excited state might still exist after 3.4 and 57 ps. In the fifth EADS (magenta line in the inset, Figure 5a), a slight positive peak is observed at $\sim 640 \mathrm{~nm}$, which is probably derived from an isomerized K-like photoproduct. Thus, the ground-state photoproduct is formed after $170 \mathrm{ps}$ at the latest. In $1.7 \mu \mathrm{s}$, all transient signals disappeared without detectable formation of an M-like state, which would result from deprotonation of the protonated RSB and appearance of near-UV absorption (Figures 5a and S3). Perhaps, the initial ground state was repopulated in $1.7 \mu$ s with thermal reisomerization, ${ }^{1}$ or, more likely, considering that PR:DMAR shows weak proton pumping activity, ${ }^{14}$ an M-state was formed in $1.7 \mu \mathrm{s}$ but not detectable in our transient absorption measurements.

In view of the broad absorbance band, fs $-\mu \mathrm{s}$ transient absorption spectra were also recorded with $600 \mathrm{~nm}$ excitation and again were globally fitted in five components: $60 \mathrm{fs}, 710 \mathrm{fs}$, 43 ps, 210 ps, and 380 ns (Figure S4). Immediately after photoabsorption, the excited state was populated (gray line, Figure S4), which develops to the second EADS (red line) in 60 fs. Significantly, in the second EADS, broad negative signals are seen at $>\sim 620 \mathrm{~nm}$, which are assigned to a mixture of GSB and SE. Moreover, in contrast to the $500 \mathrm{~nm}$ excitation data, no negative signal was observed below $\sim 480 \mathrm{~nm}$. This observation indicates that to some extent, different ground state components of PR:DMAR were excited, by the 500 and $600 \mathrm{~nm}$ excitation. In the third and fourth EADS (blue and green lines, respectively, Figure S4), spectral features similar to the second EADS were observed, while the $>700 \mathrm{~nm}$ negative SE signal was still present. Furthermore, as in the transient absorption spectra obtained upon $500 \mathrm{~nm}$ excitation, only a tiny photoproduct at $\sim 640 \mathrm{~nm}$ was detected upon $600 \mathrm{~nm}$ excitation (magenta line, Figure S4a, inset), without detectable formation of an M-intermediate. Hence, globally, the photodynamics are quite similar upon 500 or $600 \mathrm{~nm}$ excitation. In Figure $5 \mathrm{~b}$, the photocycle of PR:DMAR is shown as we deduce from excitation at $500 \mathrm{~nm}$.

It is interesting to compare the photochemistry of PR:DMAR with that of PR:MMAR, reported previously, ${ }^{21}$ because DMAR and MMAR have very similar chemical structures, except that in MMAR, the aromatic amino substituent only contains a single methyl group. ${ }^{14}$ In PR:MMAR, three excited state decays were observed in $\sim 3$, $\sim 30$, and $\sim 300$ ps, and we proposed that the photoisomerization proceeds within $\sim 30 \mathrm{ps}^{21}$ Even though it is difficult to derive this from only the present data set, we propose that upon excitation of PR:DMAR at $500 \mathrm{~nm}$, the excited state decay proceeds in $3.4,57$, and 170 ps (Figure 5a) in view of the similar DADS and time constants in PR:MMAR. In addition, the peak position and the peak width of the K-like intermediate of PR:DMAR (magenta inset, Figure 5a) are quite comparable with the photoproduct detected in PR:MMAR. ${ }^{21}$ Considering the ratio of the peak intensities of the biggest EADS (red line in Figure 5a, top) and the photoproduct state (magenta line, Figure 5a, inset), it can be suggested that the efficiency of photoproduct formation in PR:DMAR is approximately half of that in PR:MMAR. Likely, this small photoproduct yield of PR:DMAR explains the low proton pumping activity of PR:DMAR, which is less than half of that of PR:MMAR. ${ }^{14}$

Comparison of Native A1 Retinal with Reconstituted Retinal Analogues in PR. At last, we briefly overview the photochemistry of PR:A2, PR:MOA2, and PR:DMAR, that we reported in this manuscript, together with the previously published PR:A1 photochemistry.

PR:A1: A natural light-driven proton pump, characterized by rapid and efficient trans-cis isomerization of its chromophore, recruited from retinal $\mathrm{A} 1$, subsequent rapid proton transfer from the protonated Schiff base to initiate proton transport, little energy loss by fluorescence, and a quite good pumping rate.

PR:A2: The additional double bond in the retinal moiety leads to a less flexible ring structure but effectuates a significant red shift relative to $\mathrm{Al}(\sim 30 \mathrm{~nm})$ thanks to the elongated conjugated system. This analogue maintains rapid and efficient trans-cis isomerization, but the proton transfer seems to proceed slightly slower, possibly due to slight rearrangements in the binding site structure to optimally accommodate this analogue. Nevertheless, the proton pumping rate is not strongly affected, and the action spectrum is also red-shifted. ${ }^{14}$

PR:MOA2: This analogue with a monomethyloxy substituent at the ring-C3 position fits much better than $\mathrm{DMAR}^{14}$ and evokes a very large red shift $(\sim 65 \mathrm{~nm})$, probably thanks to the elongated conjugated system in combination with the 
methyloxy substituent, that will increase the charge delocalization in the system and may induce some resonance effects. In spite of this, no photoproducts or proton transfer are observed and instead very rapid decay of the excited states to the ground state occurs. The ground state also seems to contain some 13cis chromophore, which may complicate the photodynamics and may be correlated with the observation that only under white light illumination, very low proton pump activity is observed without a clear red shift in the action spectrum. ${ }^{14}$

PR:DMAR: Because of the bulky dimethylamino substituent at the ring-C3 position, this analogue does not fit very well in the PR binding site ${ }^{14}$ and will require more substantial rearrangements. Nevertheless, it induces a quite significant red shift $(\sim 40 \mathrm{~nm})$, most likely because thanks to the substituent, it has access to resonance structures with enhanced electron delocalization, similar to MMAR. ${ }^{21,57}$ However, the rate and extent of photoisomerization are strongly reduced, and proton transfer is slowed down. This agrees with the low proton pumping rate determined experimentally. ${ }^{14}$ Nevertheless, the action spectrum is also red-shifted. ${ }^{14}$

From this overview, we may cautiously conclude that for maintaining substantial proton pumping, rearrangements in the binding site should be avoided as much as possible, unless structural modifications in the analogue can be properly offset by protein mutations. Modifications in the ring that effectively communicate with the conjugated system will be most effective in inducing red shifts in the absorbance band, but their size is an important factor, a condition which, however, probably will vary for different rhodopsins. Again, offsetting this by protein mutation should be an option. Finally, it seems that amino substituents are most effective in combining red-shifting properties with maintaining activity, probably because resonance with the Schiff base yields optimal electron delocalization and molecular orbital hybridization, which even can be further enhanced by protein mutation. ${ }^{14}$ MMAR already combines these positive elements to some extent, ${ }^{14,21,43}$ but further optimization in combination with protein modification is required, in particular to enhance pump activity. The strongly negative effect of the methoxy substituent on the pump activity is clearly due to nearly complete blockage of photoproduct formation. Possibly, electronic effects of the methoxy group affect the excited states, or it indirectly affects the conformation of the counterions-water-Schiff base complex by rearranging the protein structure around the ring. A recent theoretical study indicates that substituents on the conjugated chain may affect the mixing and lifetime of excited states and the speed of photoisomerization. ${ }^{58}$ This may relate to the negative effects of the methoxy and (di)methylamino substituents on the photoisomerization efficiency in PR:MOA2, PR:DMAR, and PR:MMAR, respectively, the stronger electronegative methoxy group exerting the most inhibitory effect. Further biophysical and theoretical studies should be able to analyze these systems in more detail.

\section{CONCLUSIONS}

Insights into the photodynamics of microbial rhodopsins containing retinal analogues that red-shift their absorbance band is very limited; however, it is of high importance for further developments in fields such as optogenetics and fluorescent voltage sensors. In this study, we present a comparison of the photochemistry of three red-shifted analogues of the proton pump PR using femto- to submilli- second transient absorption spectroscopy. The PR analogue pigments contain the retinal analogues A2, MOA2, and DMAR, respectively, as their chromophore. This red-shifts the absorbance band by about 30,60 , and $40 \mathrm{~nm}$, respectively, relative to the native retinal $\mathrm{A} 1 .^{14}$ In $\mathrm{PR}: \mathrm{A} 2$, the photoisomerization proceeds with $1.4,3.0$, and/or 13 ps time constants upon $510 \mathrm{~nm}$ excitation at $\mathrm{pH} 7.0$, which is comparable to that in native PR:A1. The additional $\mathrm{C} 3=\mathrm{C} 4$ double bond in A2 (Figure 1) apparently does not strongly influence the photodynamics. Moreover, M-intermediate formation, reflecting deprotonation of the protonated Schiff base linkage to the protein, predominantly occurred with the $67 \mu$ s constant, which is significantly slower than that of PR:A1. Notably, green $(510 \mathrm{~nm})$ and red $(620 \mathrm{~nm})$ excitation resulted in different excited state properties, but the photoproduct spectra were very similar. We infer that $510 \mathrm{~nm}$ photons can trigger the photocycle more efficiently than 620 $\mathrm{nm}$ photons. On the other hand, photoisomerization was not detectable in PR:MOA2. All excited molecules relaxed to the initial ground state with time constants of 2.0 and $4.2 \mathrm{ps}$, without detectable photoproduct formation. Finally, the photoproduct was detected in PR:DMAR but only in small quantities. The excited state transition kinetics obtained with PR:DMAR (3.4, 57 and 170 ps) were similar to those in PR:MMAR, reported before. ${ }^{21}$ Moreover, it is suggested that different ground-state species were excited at 500 and $600 \mathrm{~nm}$ excitations, implying that ground-state heterogeneity exists in PR:DMAR at $\mathrm{pH}$ 9.0. The transient absorption signals completely decayed in $1.7 \mu$ s without detectable formation of an M-like state in PR:DMAR, probably implying that the Mstate signal was too weak to be detected in our measurements. Overall, the photodynamical signature of these PR analogue pigments correlates well with their functional activity as proton pumps.

\section{ASSOCIATED CONTENT}

\section{Supporting Information}

The Supporting Information is available free of charge on the ACS Publications website at DOI: 10.1021/acs.jpcb.9b01136.

Additional femto- to submillisecond transient absorption data with selected time traces (PDF)

\section{AUTHOR INFORMATION}

\section{Corresponding Author}

*E-mail: j.t.m.kennis@vu.nl.

ORCID $\odot$

Yusaku Hontani: 0000-0001-8853-9454 Srividya Ganapathy: 0000-0003-1264-9387

Willem J. de Grip: 0000-0001-7637-4920

John T.M. Kennis: 0000-0002-3563-2353

\section{Notes}

The authors declare no competing financial interest.

\section{ACKNOWLEDGMENTS}

Y.H. and J.T.M.K. were supported by the Chemical Sciences Council of the Netherlands Organization for Scientific Research (NWO-CW) through a VICI grant to J.T.M.K., and a Middelgroot investment grant to J.T.M.K. S.G. and W.J.d.G. were supported by Leiden University and the research programme of BioSolar Cells (BSC Core Project Grant C2.9), cofinanced by the Dutch Ministry of Economic Affairs. 


\section{REFERENCES}

(1) Ernst, O. P.; Lodowski, D. T.; Elstner, M.; Hegemann, P.; Brown, L. S.; Kandori, H. Microbial and Animal Rhodopsins: Structures, Functions, and Molecular Mechanisms. Chem. Rev. 2014, 114, 126-163.

(2) Grote, M.; Engelhard, M.; Hegemann, P. Of Ion Pumps, Sensors and Channels - Perspectives on Microbial Rhodopsins between Science and History. Biochim. Biophys. Acta, Bioenerg. 2014, 1837, $533-545$.

(3) Boyden, E. S.; Zhang, F.; Bamberg, E.; Nagel, G.; Deisseroth, K. Millisecond-Timescale, Genetically Targeted Optical Control of Neural Activity. Nat. Neurosci. 2005, 8, 1263-1268.

(4) Mohammad, F.; Stewart, J. C.; Ott, S.; Chlebikova, K.; Chua, J. Y.; Koh, T.-W.; Ho, J.; Claridge-Chang, A. Optogenetic Inhibition of Behavior with Anion Channelrhodopsins. Nat. Methods 2017, 14, 271-274.

(5) Kato, H. E.; Inoue, K.; Abe-Yoshizumi, R.; Kato, Y.; Ono, H.; Konno, M.; Hososhima, S.; Ishizuka, T.; Hoque, M. R.; Kunitomo, $\mathrm{H}$.; et al. Structural basis for $\mathrm{Na}+$ transport mechanism by a lightdriven Na+ pump. Nature 2015, 521, 48-53.

(6) Chow, B. Y.; Han, X.; Dobry, A. S.; Qian, X.; Chuong, A. S.; Li, M.; Henninger, M. A.; Belfort, G. M.; Lin, Y.; Monahan, P. E.; et al. High-Performance Genetically Targetable Optical Neural Silencing by Light-Driven Proton Pumps. Nature 2010, 463, 98-102.

(7) Kralj, J. M.; Hochbaum, D. R.; Douglass, A. D.; Cohen, A. E. Electrical Spiking in Escherichia Coli Probed with a Fluorescent Voltage-Indicating Protein. Science 2011, 333, 345-348.

(8) Kralj, J. M.; Douglass, A. D.; Hochbaum, D. R.; MacLaurin, D.; Cohen, A. E. Optical Recording of Action Potentials in Mammalian Neurons Using a Microbial Rhodopsin. Nat. Methods 2012, 9, 90-95.

(9) Hochbaum, D. R.; Zhao, Y.; Farhi, S. L.; Klapoetke, N.; Werley, C. A.; Kapoor, V.; Zou, P.; Kralj, J. M.; MacLaurin, D.; SmedemarkMargulies, N.; et al. All-Optical Electrophysiology in Mammalian Neurons Using Engineered Microbial Rhodopsins. Nat. Methods 2014, 11, 825-833.

(10) Weissleder, R.; Ntziachristos, V. Shedding Light onto Live Molecular Targets. Nat. Med. 2003, 9, 123-128.

(11) Sineshchekov, O. A.; Govorunova, E. G.; Wang, J.; Spudich, J. L. Enhancement of the Long-Wavelength Sensitivity of Optogenetic Microbial Rhodopsins by 3,4-Dehydroretinal. Biochemistry 2012, 51, 4499-4506.

(12) Azimihashemi, N.; Erbguth, K.; Vogt, A.; Riemensperger, T.; Rauch, E.; Woodmansee, D.; Nagpal, J.; Brauner, M.; Sheves, M.; Fiala, A.; et al. Synthetic Retinal Analogues Modify the Spectral and Kinetic Characteristics of Microbial Rhodopsin Optogenetic Tools. Nat. Commun. 2014, 5, 5810.

(13) Ganapathy, S.; Bécheau, O.; Venselaar, H.; Frölich, S.; van der Steen, J. B.; Chen, Q.; Radwan, S.; Lugtenburg, J.; Hellingwerf, K. J.; de Groot, H. J. M.; et al. Modulation of Spectral Properties and Pump Activity of Proteorhodopsins by Retinal Analogues. Biochem. J. 2015, 467, 333-343.

(14) Ganapathy, S.; Venselaar, H.; Chen, Q.; De Groot, H. J. M.; Hellingwerf, K. J.; De Grip, W. J. Retinal-Based Proton Pumping in the Near Infrared. J. Am. Chem. Soc. 2017, 139, 2338-2344.

(15) Herwig, L.; Rice, A. J.; Bedbrook, C. N.; Zhang, R. K.; Lignell, A.; Cahn, J. K. B.; Renata, H.; Dodani, S. C.; Cho, I.; Cai, L.; et al. Directed Evolution of a Bright Near-Infrared Fluorescent Rhodopsin Using a Synthetic Chromophore. Cell Chem. Biol. 2017, 24, 415-425.

(16) Shen, Y.-C.; Sasaki, T.; Matsuyama, T.; Yamashita, T.; Shichida, Y.; Okitsu, T.; Yamano, Y.; Wada, A.; Ishizuka, T.; Yawo, H.; et al. Red-Tuning of the Channelrhodopsin Spectrum Using Long Conjugated Retinal Analogues. Biochemistry 2018, 57, 24.

(17) Wald, G. The Molecular Basis of Visual Excitation. Nature 1968, 219, 800-807.

(18) Foster, R. G.; Garcia-Fernandez, J. M.; Provencio, I.; DeGrip, W. J. Opsin Localization and Chromophore Retinoids Identified within the Basal Brain of the Lizard Anolis Carolinensis. J. Comp. Physiol., A 1993, 172, 33-45.
(19) Jokela-Määttä, M.; Pahlberg, J.; Lindström, M.; Zak, P. P.; Porter, M.; Ostrovsky, M. A.; Cronin, T. W.; Donner, K. Visual Pigment Absorbance and Spectral Sensitivity of the Mysis Relicta Species Group (Crustacea, Mysida) in Different Light Environments. J. Comp. Physiol., A 2005, 191, 1087-1097.

(20) Yizhar, O.; Fenno, L. E.; Davidson, T. J.; Mogri, M.; Deisseroth, K. Optogenetics in Neural Systems. Neuron 2011, 71, 9-34.

(21) Hontani, Y.; Ganapathy, S.; Frehan, S.; Kloz, M.; de Grip, W. J.; Kennis, J. T. M. Strong PH-Dependent Near-Infrared Fluorescence in a Microbial Rhodopsin Reconstituted with a Red-Shifting Retinal Analogue. J. Phys. Chem. Lett. 2018, 9, 6469-6474.

(22) Kloz, M.; Weißenborn, J.; Polívka, T.; Frank, H. A.; Kennis, J. T. M. Spectral watermarking in femtosecond stimulated Raman spectroscopy: resolving the nature of the carotenoid S* state. Phys. Chem. Chem. Phys. 2016, 18, 14619-14628.

(23) Hontani, Y.; Inoue, K.; Kloz, M.; Kato, Y.; Kandori, H.; Kennis, J. T. M. The Photochemistry of Sodium Ion Pump Rhodopsin Observed by Watermarked Femto- to Submillisecond Stimulated Raman Spectroscopy. Phys. Chem. Chem. Phys. 2016, 18, 2472924736.

(24) Hontani, Y.; Kloz, M.; Polívka, T.; Shukla, M. K.; Sobotka, R.; Kennis, J. T. M. Molecular Origin of Photoprotection in Cyanobacteria Probed by Watermarked Femtosecond Stimulated Raman Spectroscopy. J. Phys. Chem. Lett. 2018, 9, 1788-1792.

(25) Hontani, Y.; Marazzi, M.; Stehfest, K.; Mathes, T.; Van Stokkum, I. H. M.; Elstner, M.; Hegemann, P.; Kennis, J. T. M. Reaction Dynamics of the Chimeric Channelrhodopsin. Sci. Rep. 2017, 7, 7217.

(26) Ravensbergen, J.; Abdi, F. F.; Van Santen, J. H.; Frese, R. N.; Dam, B.; Van De Krol, R.; Kennis, J. T. M. Unraveling the Carrier Dynamics of BiVO4: A Femtosecond to Microsecond Transient Absorption Study. J. Phys. Chem. C 2014, 118, 27793-27800.

(27) Alexandre, M. T. A.; Domratcheva, T.; Bonetti, C.; Van Wilderen, L. J. G. W.; Van Grondelle, R.; Groot, M.-L.; Hellingwerf, K. J.; Kennis, J. T. M. Primary Reactions of the LOV2 Domain of Phototropin Studied with Ultrafast Mid-Infrared Spectroscopy and Quantum Chemistry. Biophys. J. 2009, 97, 227-237.

(28) Snellenburg, J. J.; Laptenok, S. P.; Seger, R.; Mullen, K. M.; van Stokkum, I. H. M. Glotaran : A Java-Based Graphical User Interface for the R Package TIMP. J. Stat. Softw. 2012, 49, 1-22.

(29) Van Stokkum, I. H. M.; Larsen, D. S.; Van Grondelle, R. Global and Target Analysis of Time-Resolved Spectra. Biochim. Biophys. Acta, Bioenerg. 2004, 1657, 82-104.

(30) Hontani, Y.; Broser, M.; Silapetere, A.; Krause, B. S.; Hegemann, P.; Kennis, J. T. M. The Femtosecond-to-Second Photochemistry of Red-Shifted Fast-Closing Anion Channelrhodopsin PsACR1. Phys. Chem. Chem. Phys. 2017, 19, 30402-30409.

(31) Kennis, J. T.; Groot, M.-L. Ultrafast Spectroscopy of Biological Photoreceptors. Curr. Opin. Struct. Biol. 2007, 17, 623-630.

(32) Toh, K. C.; Stojković, E. A.; Van Stokkum, I. H. M.; Moffat, K.; Kennis, J. T. M. Fluorescence Quantum Yield and Photochemistry of Bacteriophytochrome Constructs. Phys. Chem. Chem. Phys. 2011, 13, 11985-11997.

(33) Dioumaev, A. K.; Brown, L. S.; Shih, J.; Spudich, E. N.; Spudich, J. L.; Lanyi, J. K. Proton Transfers in the Photochemical Reaction Cycle of Proteorhodopsin $\dagger$. Biochemistry 2002, 41, 53485358.

(34) Krebs, R. A.; Dunmire, D.; Partha, R.; Braiman, M. S. Resonance Raman Characterization of Proteorhodopsin's Chromophore Environment. J. Phys. Chem. B 2003, 107, 7877-7883.

(35) Smith, S. O.; Braiman, M. S.; Myers, A. B.; Pardoen, J. A.; Courtin, J. M. L.; Winkel, C.; Lugtenburg, J.; Mathies, R. A. Vibrational Analysis of the All-Trans-Retinal Chromophore in LightAdapted Bacteriorhodopsin. J. Am. Chem. Soc. 1987, 109, 3108-3125.

(36) Bergo, V.; Amsden, J. J.; Spudich, E. N.; Spudich, J. L.; Rothschild, K. J. Structural Changes in the Photoactive Site of Proteorhodopsin during the Primary Photoreaction $\dagger$. Biochemistry 2004, 43, 9075-9083. 
(37) Smith, S. O.; Pardoen, J. A.; Lugtenburg, J.; Mathies, R. A. Vibrational Analysis of the 13-Cis-Retinal Chromophore in DarkAdapted Bacteriorhodopsin. J. Phys. Chem. 1987, 91, 804-819.

(38) Imasheva, E. S.; Shimono, K.; Balashov, S. P.; Wang, J. M.; Zadok, U.; Sheves, M.; Kamo, N.; Lanyi, J. K. Formation of a LongLived Photoproduct with a Deprotonated Schiff Base in Proteorhodopsin, and Its Enhancement by Mutation of Asp227 $\dagger$. Biochemistry 2005, 44, 10828-10838.

(39) Pfleger, N.; Lorch, M.; Woerner, A. C.; Shastri, S.; Glaubitz, C. Characterisation of Schiff Base and Chromophore in Green Proteorhodopsin by Solid-State NMR. J. Biomol. NMR 2008, 40, $15-21$.

(40) Doukas, A. G.; Aton, B.; Callender, R. H.; Ebrey, T. G. Resonance Raman Studies of Bovine Metarhodopsin I and Metarhodopsin II. Biochemistry 1978, 17, 2430-2435.

(41) Ogren, J. I.; Mamaev, S.; Russano, D.; Li, H.; Spudich, J. L.; Rothschild, K. J. Retinal Chromophore Structure and Schiff Base Interactions in Red-Shifted Channelrhodopsin-1 from Chlamydomonas Augustae. Biochemistry 2014, 53, 3961-3970.

(42) Kajimoto, K.; Kikukawa, T.; Nakashima, H.; Yamaryo, H.; Saito, Y.; Fujisawa, T.; Demura, M.; Unno, M. Transient Resonance Raman Spectroscopy of a Light-Driven Sodium-Ion-Pump Rhodopsin from Indibacter Alkaliphilus. J. Phys. Chem. B 2017, 121, 4431-4437.

(43) Mei, G.; Mamaeva, N.; Ganapathy, S.; Wang, P.; DeGrip, W. J.; Rothschild, K. J. Raman Spectroscopy of a near Infrared Absorbing Proteorhodopsin: Similarities to the Bacteriorhodopsin O Photointermediate. PLoS One 2018, 13, No. e0209506.

(44) Lenz, M. O.; Huber, R.; Schmidt, B.; Gilch, P.; Kalmbach, R.; Engelhard, M.; Wachtveitl, J. First Steps of Retinal Photoisomerization in Proteorhodopsin. Biophys. J. 2006, 91, 255-262.

(45) Mehler, M.; Scholz, F.; Ullrich, S. J.; Mao, J.; Braun, M.; Brown, L. J.; Brown, R. C. D.; Fiedler, S. A.; Becker-Baldus, J.; Wachtveitl, J.; et al. The EF Loop in Green Proteorhodopsin Affects Conformation and Photocycle Dynamics. Biophys. J. 2013, 105, 385-397.

(46) Verhoefen, M.-K.; Bamann, C.; Blöcher, R.; Förster, U.; Bamberg, E.; Wachtveitl, J. The Photocycle of Channelrhodopsin-2: Ultrafast Reaction Dynamics and Subsequent Reaction Steps. ChemPhysChem 2010, 11, 3113-3122.

(47) Scholz, F.; Bamberg, E.; Bamann, C.; Wachtveitl, J. Tuning the Primary Reaction of Channelrhodopsin-2 by Imidazole, PH, and SiteSpecific Mutations. Biophys. J. 2012, 102, 2649-2657.

(48) Huber, R.; Köhler, T.; Lenz, M. O.; Bamberg, E.; Kalmbach, R.; Engelhard, M.; Wachtveitl, J. pH-Dependent Photoisomerization of Retinal in Proteorhodopsin $\dagger$. Biochemistry 2005, 44, 1800-1806.

(49) Rupenyan, A.; Van Stokkum, I. H. M.; Arents, J. C.; Van Grondelle, R.; Hellingwerf, K. J.; Groot, M. L. Reaction Pathways of Photoexcited Retinal in Proteorhodopsin Studied by Pump-DumpProbe Spectroscopy. J. Phys. Chem. B 2009, 113, 16251-16256.

(50) Köhler, T.; Weber, I.; Glaubitz, C.; Wachtveitl, J. Proteorhodopsin Photocycle Kinetics Between PH 5 and PH 9. Photochem. Photobiol. 2017, 93, 762-771.

(51) Mathies, R.; Brito Cruz, C.; Pollard, W.; Shank, C. Direct Observation of the Femtosecond Excited-State Cis-Trans Isomerization in Bacteriorhodopsin. Science 1988, 240, 777-779.

(52) Kandori, H.; Yoshihara, K.; Tomioka, H.; Sasabe, H. Primary Photochemical Events in Halorhodopsin Studied by Subpicosecond Time-Resolved Spectroscopy. J. Phys. Chem. 1992, 96, 6066-6071.

(53) Tahara, S.; Takeuchi, S.; Abe-Yoshizumi, R.; Inoue, K.; Ohtani, H.; Kandori, H.; Tahara, T. Ultrafast Photoreaction Dynamics of a Light-Driven Sodium-Ion-Pumping Retinal Protein from Krokinobacter Eikastus Revealed by Femtosecond Time-Resolved Absorption Spectroscopy. J. Phys. Chem. Lett. 2015, 6, 4481-4486.

(54) Shibata, M.; Yoshitsugu, M.; Mizuide, N.; Ihara, K.; Kandori, H. Halide Binding by the D212N Mutant of Bacteriorhodopsin Affects Hydrogen Bonding of Water in the Active Site $\dagger$. Biochemistry 2007, $46,7525-7535$.

(55) Váró, G.; Brown, L. S.; Lakatos, M.; Lanyi, J. K. Characterization of the Photochemical Reaction Cycle of Proteorhodopsin. Biophys. J. 2003, 84, 1202-1207.
(56) Bruun, S.; Stoeppler, D.; Keidel, A.; Kuhlmann, U.; Luck, M.; Diehl, A.; Geiger, M.-A.; Woodmansee, D.; Trauner, D.; Hegemann, P.; et al. Light-Dark Adaptation of Channelrhodopsin Involves Photoconversion between the All-Trans and 13-Cis Retinal Isomers. Biochemistry 2015, 54, 5389-5400.

(57) Ganapathy, S.; Kratz, S.; Chen, Q.; Hellingwerf, K. J.; de Groot, H. J. M.; Rothschild, K. J.; de Grip, W. J. Red-Shifted and Nearinfrared Active Analog Pigments Based upon Archaerhodopsin-3. Photochem. Photobiol. 2019, DOI: 10.1111/php.13093.

(58) Manathunga, M.; Yang, X.; Olivucci, M. Electronic State Mixing Controls the Photoreactivity of a Rhodopsin with all-trans Chromophore Analogues. J. Phys. Chem. Lett. 2018, 9, 6350-6355. 